\title{
THE SIMPLICIAL VOLUME OF 3-MANIFOLDS WITH BOUNDARY
}

\author{
MICHELLE BUCHER, ROBERTO FRIGERIO, AND CRISTINA PAGLIANTINI
}

\begin{abstract}
We provide sharp lower bounds for the simplicial volume of compact 3-manifolds in terms of the simplicial volume of their boundaries. As an application, we compute the simplicial volume of several classes of 3-manifolds, including handlebodies and products of surfaces with the interval. Our results provide the first exact computation of the simplicial volume of a compact manifold whose boundary has positive simplicial volume. We also compute the minimal number of tetrahedra in a (loose) triangulation of the product of a surface with the interval.
\end{abstract}

\section{INTRODUCTION}

The simplicial volume is an invariant of manifolds introduced by Gromov in his seminal paper Gro82. If $M$ is a connected, compact, oriented manifold with (possibly empty) boundary, then the simplicial volume of $M$ is the infimum of the sum of the absolute values of the coefficients over all singular chains representing the real fundamental cycle of $M$ (see Section 1). It is usually denoted by $\|M\|$ if $M$ is closed, and by $\|M, \partial M\|$ if $\partial M \neq \emptyset$. If $M$ is open, the fundamental class and the simplicial volume of $M$ admit analogous definitions in the context of homology of locally finite chains, but in this paper we will restrict our attention to compact manifolds: unless otherwise stated, henceforth every manifold is assumed to be compact. Observe that the simplicial volume of an oriented manifold does not depend on its orientation and that it naturally extends also to nonorientable or disconnected manifolds.

Even if it depends only on the homotopy type of a manifold, the simplicial volume is deeply related to the geometric structures that a manifold can carry. For example, closed manifolds which support negatively curved Riemannian metrics have nonvanishing simplicial volume, while the simplicial volume of flat or spherical manifolds is null (see e.g. Gro82]). Several vanishing and nonvanishing results for the simplicial volume are available by now, but the exact value of nonvanishing simplicial volumes is known only in a very few cases. If $M$ is (the natural compactification of) a complete finite-volume hyperbolic $n$-manifold without boundary, then a celebrated result by Gromov and Thurston implies that the simplicial volume of $M$

Michelle Bucher was supported by Swiss National Science Foundation project PP00P2128309/1. The authors thank the Institute Mittag-Leffler in Djursholm, Sweden, for their warm hospitality during the preparation of this paper. 
is equal to the Riemannian volume of $M$ divided by the volume $v_{n}$ of the regular ideal geodesic $n$-simplex in hyperbolic space (see [Gro82, Thu79] for the compact case and e.g. [Fra04, FP10, FM11, BBI13 for the cusped case). The only other exact computation of nonvanishing simplicial volume is for the product of two closed hyperbolic surfaces or more generally manifolds locally isometric to the product of two hyperbolic planes BK08. Building on these examples, more values for the simplicial volume can be obtained by surgery or by taking connected sums or amalgamated sums over submanifolds with amenable fundamental group (see e.g. [Gro82, Kue03, $\left.\mathrm{BBF}^{+} 14\right]$ ) however not by taking products.

For hyperbolic manifolds with geodesic boundary, it is proved by Jungreis [Jun97] that if $M$ is such a manifold and $\partial M \neq \emptyset$, then $\|M, \partial M\|$ strictly exceeds $\operatorname{Vol}(M) / v_{n}$, and the last two authors showed that there exist, in any dimension, examples for which $\operatorname{Vol}(M) /\|M, \partial M\|$ is arbitrarily close to $v_{n}$ [FP10. These results were the sharpest estimates so far for the simplicial volume of manifolds whose boundary has positive simplicial volume. We provide here the first exact computations of $\|M, \partial M\|$ for classes of 3-manifolds for which $\|\partial M\|>0$.

The simplicial volume of 3-manifolds with boundary. If $M$ is a connected oriented $n$-manifold with boundary, then the usual boundary map takes any relative fundamental cycle of $M$ to the sum of fundamental cycles of the components of $\partial M$. As a consequence, for any $n$-manifold $M$ we have

$$
\|M, \partial M\| \geq \frac{\|\partial M\|}{n+1} .
$$

In particular, if $\|\partial M\|>0$, then $\|M, \partial M\|>0$.

The main result of this paper concerns 3-dimensional manifolds, and provides a sharp lower bound for $\|M, \partial M\|$ in terms of $\|\partial M\|$ :

Theorem 1. Let $M$ be a 3-manifold. Then there is a sharp inequality

$$
\|M, \partial M\| \geq \frac{3}{4}\|\partial M\| .
$$

The fact that the bound of Theorem 1 is sharp is an immediate consequence of Theorem 2. Theorems 1 and 2 are proved in Section 2 . We will see in Theorem 4 that in the case of a boundary irreducible aspherical 3 -manifold, the constant $3 / 4$ can be improved to $5 / 4$.

Stable $\Delta$-complexity and simplicial volume. If $M$ is an $n$-manifold, we denote by $\sigma(M)$ the $\Delta$-complexity of $M$, i.e. the minimal number of top dimensional simplices in a triangulation of $M$. We employ here the word "triangulation" in a loose sense, as is customary in geometric topology: a triangulation is the realization of $M$ as the gluing of finitely many $n$-simplices via some simplicial pairing of their codimension- 1 faces. It is easy to see that the inequality $\|M, \partial M\| \leq \sigma(M)$ holds (see e.g. [FFM12, Proposition 0.1] or the discussion in the proof of Theorem 5 in Section 3 ). 
The simplicial volume is multiplicative with respect to finite coverings, while for every degree $d$ covering $\widehat{M} \stackrel{d}{\rightarrow} M$ we have

$$
\sigma(\widehat{M}) \leqslant d \cdot \sigma(M)
$$

which is very often a strict inequality. The stable $\Delta$-complexity $\sigma_{\infty}(M)$ of $M$ is defined by setting

$$
\sigma_{\infty}(M)=\inf _{\widehat{M} \stackrel{d}{\rightarrow} M}\left\{\frac{\sigma(\widehat{M})}{d}\right\}
$$

where the infimum is taken over all finite coverings $\widehat{M} \stackrel{d}{\rightarrow} M$ of any finite degree $d$. The definition of the stable $\Delta$-complexity, which was introduced by Milnor and Thurston in [MT77, is made to be multiplicative with respect to finite coverings. The inequality $\|M, \partial M\| \leq \sigma(M)$ and the multiplicativity of the simplicial volume with respect to finite coverings imply that

$$
\|M, \partial M\| \leq \sigma_{\infty}(M)
$$

for every $n$-manifold $M$. It has recently been established [FFM12] that this inequality is strict for closed hyperbolic manifolds of dimension $\geq 4$.

The simplicial volume of handlebodies. Every Seifert manifold with nonempty boundary has a finite covering which is the product of a surface with a circle. Such a covering admits in turn nontrivial self-coverings, and has therefore null stable $\Delta$-complexity. As a consequence, for every Seifert manifold with nonempty boundary both the stable $\Delta$-complexity and the simplicial volume vanish. In particular, the inequality (2) is an equality. (The same is true for closed Seifert manifolds with infinite fundamental group.) Non zero examples where the simplicial volume equals the stable $\Delta$-complexity are provided by the following result.

Theorem 2. Let $M$ be a Seifert manifold with nonempty boundary, and let $N$ be obtained by performing a finite number of 1-handle additions on $M$. Then

$$
\|N, \partial N\|=\sigma_{\infty}(N)=\frac{3}{4}\|\partial N\| .
$$

For every $g \in \mathbb{N}$ let us denote by $H_{g}$ the orientable handlebody of genus g. We easily have $\left\|H_{0}, \partial H_{0}\right\|=\left\|H_{1}, \partial H_{1}\right\|=0$. Since $H_{1}$ is a Seifert manifold and $H_{g}$ can be obtained by performing $g-1$ handle additions on $H_{1}$, Theorem 2 implies the following:

Corollary 3. For every $g \geq 2$, the equalities

$$
\left\|H_{g}, \partial H_{g}\right\|=\sigma_{\infty}\left(H_{g}\right)=\frac{3}{4}\left\|\partial H_{g}\right\|=3(g-1)
$$

hold. 
This improves the bounds

$$
\frac{4}{3}(g-1) \leq\left\|H_{g}, \partial H_{g}\right\| \leq \sigma_{\infty}\left(H_{g}\right) \leq 3(g-1)
$$

exhibited by Kuessner Kue03. Note that the upper bound also follows from the computation of the $\Delta$-complexity of the handlebody $\sigma\left(H_{g}\right)=3 g-2$ established by Jaco and Rubinstein [JR].

Aspherical manifolds with $\pi_{1}$-injective boundary. Recall that a connected manifold $M$ is aspherical if $\pi_{i}(M)=0$ for every $i \geq 2$, or, equivalently, if the universal covering of $M$ is contractible. If $M$ is disconnected, we say that $M$ is aspherical if every connected component of $M$ is. Moreover, we say that $M$ is boundary irreducible if for every connected component $B$ of $\partial M$ the inclusion $B \hookrightarrow M$ induces an injective map on fundamental groups (we borrow this terminology from the context of 3-manifold topology, and use it also in the higher dimensional case).

The estimate provided by Theorem 1 may be improved in the case of boundary irreducible aspherical 3-manifolds. More precisely, we prove the following:

Theorem 4. Let $M$ be a boundary irreducible aspherical 3-manifold. Then there is a sharp inequality

$$
\|M, \partial M\| \geq \frac{5}{4}\|\partial M\|
$$

The equality is realized by products of surfaces with intervals (Corollary 6) for which we first compute the $\Delta$-complexity. Both theorems and their corollary will be proven in Section 3 .

Theorem 5. Let $S_{g}$ be a closed orientable surface of genus $g \geq 1$ and let $M_{g}=S_{g} \times[0,1]$. Then

$$
\sigma\left(M_{g}\right)=10(g-1)+6
$$

This result has recently also been obtained by Jaco, Johnson, Spreer and Tillmann using different methods [JJST]. There are remarkably few examples of exact computations of $\Delta$-complexity of manifolds. The first family of examples is given by surfaces, Jaco, Rubinstein and Tillmann computed the $\Delta$-complexity of an infinite family of lens spaces [JRT09, and the $\Delta$-complexity of handlebodies is computed by Jaco and Rubinstein [JR]. Moreover, a census of closed 3-manifolds up to $\Delta$-complexity 9 and 10 may be deduced from the results in [MP01] and [Mar06]. Our Theorem 5 provides the exact computation of $\Delta$-complexity for a new infinite family of examples. It might be worth mentioning that, in the case of manifolds with boundary, the minimal number of simplices in ideal triangulations of manifolds, rather than in (loose) triangulations, has been computed for several families of 3-manifolds. 
Corollary 6. Let $S_{g}$ be a closed orientable surface of genus $g \geq 2$ and let $M_{g}=S_{g} \times[0,1]$. Then

$$
\left\|M_{g}, \partial M_{g}\right\|=\sigma_{\infty}\left(M_{g}\right)=\frac{5}{4}\left\|\partial M_{g}\right\|
$$

\section{Simplicial volume}

Let $X$ be a topological space and $Y \subseteq X$ a (possibly empty) subspace of $X$. Let $R$ be a normed ring. In this paper only the cases $R=\mathbb{R}, \mathbb{Q}$ or $\mathbb{Z}$ are considered, where each of these rings is endowed with the norm given by the absolute value. For $i \in \mathbb{N}$ we denote by $S_{i}(X)$ the set of singular $i$-simplices in $X$, by $C_{i}(X ; R)$ the module of singular $i$-chains over $R$, and we set as usual $C_{i}(X, Y ; R)=C_{i}(X ; R) / C_{i}(Y ; R)$. We observe that the $R$-module $C_{i}(X, Y ; R)$ is free and admits the preferred basis given by the classes of the singular simplices whose image is not contained in $Y$. Therefore, we will often identify $C_{i}(X, Y ; R)$ with the free $R$-module generated by $S_{i}(X) \backslash$ $S_{i}(Y)$. In particular, for $z \in C_{i}(X, Y ; R)$, it will be understood from the equality $z=\sum_{k=1}^{n} a_{k} \sigma_{k}$ that $\sigma_{k} \neq \sigma_{h}$ for $k \neq h$, and $\sigma_{k} \notin S_{i}(Y)$ for every $k$. We denote by $H_{*}(X, Y ; R)$ the singular homology of the pair $(X, Y)$ with coefficients in $R$, i.e. the homology of the complex $\left(C_{*}(X, Y ; R), d_{*}\right)$, where $d_{*}$ is the usual differential.

We endow the $R$-module $C_{i}(X, Y ; R)$ with the $L^{1}$-norm defined by

$$
\left\|\sum_{\sigma} a_{\sigma} \sigma\right\|_{R}=\sum_{\sigma}\left|a_{\sigma}\right|,
$$

where $\sigma$ ranges over the simplices in $S_{i}(X) \backslash S_{i}(Y)$. We denote simply by $\|\cdot\|$ the norm $\|\cdot\|_{\mathbb{R}}$. The norm $\|\cdot\|_{R}$ descends to a seminorm on $H_{*}(X, Y ; R)$, which is still denoted by $\|\cdot\|_{R}$ and is defined as follows: if $[\alpha] \in H_{i}(X, Y ; R)$, then

$$
\|[\alpha]\|_{R}=\inf \left\{\|\beta\|_{R}, \beta \in C_{i}(X, Y ; R), d \beta=0,[\beta]=[\alpha]\right\} .
$$

Note that although $\|\cdot\|_{\mathbb{Z}}$ is often called a seminorm in the literature, it is technically not so as it is not multiplicative in general. The real singular homology module $H_{*}(X, Y ; \mathbb{R})$ and the seminorm on $H_{*}(X, Y ; \mathbb{R})$ will be simply denoted by $H_{*}(X, Y)$ and $\|\cdot\|$ respectively.

If $M$ is a connected oriented $n$-manifold with (possibly empty) boundary $\partial M$, then we denote by $[M, \partial M]_{R}$ the fundamental class of the pair $(M, \partial M)$ with coefficients in $R$. The following definition is due to Gromov [Gro82, Thu79]:

Definition 1.1. The simplicial volume of $M$ is

$$
\|M, \partial M\|=\left\|[M, \partial M]_{\mathbb{R}}\right\|=\left\|[M, \partial M]_{\mathbb{R}}\right\|_{\mathbb{R}} .
$$

The rational, respectively integral, simplicial volume of $M$ is defined as $\|M, \partial M\|_{\mathbb{Q}}=\left\|[M, \partial M]_{\mathbb{Q}}\right\|_{\mathbb{Q}}$, resp. $\|M, \partial M\|_{\mathbb{Z}}=\left\|[M, \partial M]_{\mathbb{Z}}\right\|_{\mathbb{Z}}$. 
Just as in the real case, the rational and the integral simplicial volume may be defined also when $M$ is disconnected or nonorientable. Of course we have the inequalities $\|M, \partial M\| \leq\|M, \partial M\|_{\mathbb{Q}} \leq\|M, \partial M\|_{\mathbb{Z}}$. Using that $\mathbb{Q}$ is dense in $\mathbb{R}$, it may be shown in fact that $\|M, \partial M\|=\|M, \partial M\|_{\mathbb{Q}}$.

The integral simplicial volume does not behave as nicely as the rational or real simplicial volume. For example, it follows from the definition that $\|M\|_{\mathbb{Z}} \geq 1$ for every manifold $M$. Therefore, the integral simplicial volume cannot be multiplicative with respect to finite coverings (otherwise it should vanish on manifolds that admit finite nontrivial self-coverings, as $\left.S^{1}\right)$. Nevertheless, we will use integral cycles extensively, as they admit a clear geometric interpretation in terms of pseudomanifolds (see Section 2). In order to follow this strategy, we need the following obvious consequence of the equality $\|M, \partial M\|_{\mathbb{Q}}=\|M, \partial M\|$.

Lemma 1.2. Let $M$ be connected and oriented, and let $\varepsilon>0$ be given. Then, there exists an integral cycle $z \in C_{n}(M, \partial M ; \mathbb{Z})$ such that

$$
\frac{\|z\|_{\mathbb{Z}}}{d} \leq\|M, \partial M\|+\varepsilon
$$

where $[z]=d \cdot[M, \partial M]_{\mathbb{Z}}$ and $d>0$ is an integer.

Moreover, the boundary of a fundamental cycle for $M$ is equal to the sum of one fundamental cycle for each component of $\partial M$, so we also have:

Lemma 1.3. Let $M$ be connected and oriented, and let $z$ be an integral $n$-dimensional cycle such that $[z]=d \cdot[M, \partial M]_{\mathbb{Z}}$, where $d>0$ is an integer. Then

$$
\|\partial M\| \leq \frac{\|\partial z\|_{\mathbb{Z}}}{d}
$$

Finally, let us list some elementary properties of the simplicial volume which will be needed later.

Proposition 1.4 (Gro82). Let $M, N$ be connected oriented manifolds of the same dimension, and suppose that either $M, N$ are both closed, or they both have nonempty boundary. Let $f: N \rightarrow M$ be a map of degree $d$. Then

$$
\|N, \partial N\| \geq|d| \cdot\|M, \partial M\| \text {. }
$$

The following well-known result describes the simplicial volume of closed surfaces. In fact, the same statement also holds for connected surfaces with boundary.

Proposition 1.5 (Gro82]). Let $S$ be a closed surface. Then

$$
\|S\|=\max \{0,-2 \chi(S)\} .
$$

Let $S, S^{\prime}$ be (possibly disconnected) orientable surfaces without boundary. We say that $S^{\prime}$ is obtained from $S$ by an elementary tubing if $S^{\prime}$ is obtained from $S$ by removing two disjoint embedded disks and gluing an annulus to the resulting boundary components in such a way that the resulting surface is 
orientable. We say that $S^{\prime}$ is obtained by tubing from $S$ if it is obtained from $S$ via a finite sequence of elementary tubings. An immediate application of Proposition 1.5 yields the following:

Corollary 1.6. Let $S, S^{\prime}$ be (possibly disconnected) orientable surfaces without boundary, and suppose that $S^{\prime}$ is obtained from $S$ by tubing. Then

$$
\left\|S^{\prime}\right\| \geq\|S\| \text {. }
$$

Note that for elementary tubing, the inequality is strict unless the tube is attached to a sphere.

\section{Proof of Theorems 1 and 2}

Pseudomanifolds and integral cycles. Let $n \in \mathbb{N}$. An $n$-dimensional pseudomanifold $P$ consists of a finite number of copies of the standard $n$ simplex, a choice of pairs of $(n-1)$-dimensional faces of $n$-simplices such that each face appears in at most one of these pairs, and an affine identification between the faces of each pair. We allow pairs of distinct faces in the same $n$-simplex. It is orientable if orientations on the simplices of $P$ may be chosen in such a way that the affine identifications between the paired faces (endowed with the induced orientations) are all orientation-reversing. A face which does not belong to any pair of identified faces is a boundary face.

We denote by $|P|$ the topological realization of $P$, i.e. the quotient space of the union of the simplices by the equivalence relation generated by the identification maps. We say that $P$ is connected if $|P|$ is. We denote by $\partial|P|$ the image in $|P|$ of the boundary faces of $P$, and we say that $P$ is without boundary if $\partial|P|=\emptyset$.

A $k$-dimensional face of $|P|$ is the image in $|P|$ of a $k$-dimensional face of a simplex of $P$. Usually, we refer to 1-dimensional, resp. 0-dimensional faces of $P$ and $|P|$ as to edges, resp. vertices of $P$ and $|P|$.

It is well-known that, if $P$ is an $n$-dimensional pseudomanifold, $n \geq 3$, then $|P|$ does not need to be a manifold. However, the topological realization of any 2-pseudomanifold is a genuine surface, and singularities of orientable 3-dimensional pseudomanifolds can occur only at vertices (see e.g. Hat02, pages 108-109] and [Thu97, Exercise 1.3.2(b)]). Moreover, the boundary of the topological realization of an $n$-dimensional pseudomanifold $P$ is naturally the topological realization of an (orientable) $(n-1)$-pseudomanifold $\partial P$ without boundary, and an orientation of $P$ canonically induces an orientation on $\partial P$. In particular, if $P$ is orientable and 3-dimensional, then $\partial|P|$ is a finite union of orientable closed surfaces.

Let $M$ be an oriented connected $n$-dimensional manifold with (possibly empty) boundary $\partial M$. It is well-known that every integral relative cycle on $(M, \partial M)$ can be represented by a map from a suitable pseudomanifold

to $M$. Roughly speaking, let $z=\sum_{i=1}^{k} \varepsilon_{i} \sigma_{i}$ be an $n$-dimensional relative cycle in $C_{n}(M, \partial M ; \mathbb{Z})$ where $\varepsilon_{i}= \pm 1$ for every $i$ (note that here we do not assume that $\sigma_{i} \neq \sigma_{j}$ for $\left.i \neq j\right)$. Then one takes $k$ copies $\Delta_{1}^{n}, \ldots, \Delta_{k}^{n}$ of the 
standard $n$-simplex and assume that $\sigma_{i}$ is defined on $\Delta_{i}^{n}$. Two faces of the $\Delta_{i}^{n}$ 's are glued to each other if and only if the restriction to them of the corresponding $\sigma_{i}$ 's cancel each other in the expression of $\partial z$ as the sum of singular $(n-1)$-simplices. The continuous maps $\sigma_{i}$ glue up to a well-defined continuous map $f:(|P|, \partial|P|) \rightarrow(M, \partial M)$. Moreover, a suitable algebraic sum of the simplices of $P$ defines a relative cycle $z_{P} \in C_{n}(|P|, \partial|P| ; \mathbb{Z})$ such that the map $f_{*}$ induced by $f$ on integral singular chains sends $z_{P}$ in $f_{*}\left(z_{P}\right)=z \in C_{n}(M, \partial M ; \mathbb{Z})$. We refer the reader to [Hat02, pages 108-109] for the details.

Approximating real cycles via pseudomanifolds. As before, let $M$ be a connected oriented $n$-manifold. By Lemma 1.2 , the simplicial volume of $M$ can be computed from integral cycles. The following proposition shows that such cycles may be represented by $n$-pseudomanifolds with additional properties.

If $P$ is an $n$-dimensional pseudomanifold, we denote by $c(P)$ the number of $n$-simplices of $P$. If $P$ is associated to the integral cycle $z$, then $c(P)=\|z\|_{\mathbb{Z}}$ and $c(\partial P)=\|\partial z\|_{\mathbb{Z}}$.

Proposition 2.1. Let $\varepsilon>0$ be fixed, and suppose that $\|M, \partial M\|>0$. Then, there exists a relative integral $n$-cycle $z \in C_{n}(M, \partial M ; \mathbb{Z})$ with associated pseudomanifold $P$ such that the following conditions hold:

(1) $[z]=d \cdot[M, \partial M]_{\mathbb{Z}}$ in $H_{n}(M, \partial M ; \mathbb{Z})$ for some integer $d>0$, and

$$
\frac{\|z\|_{\mathbb{Z}}}{d} \leq\|M, \partial M\|+\varepsilon
$$

(2) $P$ is connected;

(3) every $n$-simplex of $P$ has at most $n-1$ boundary faces, and in particular

$$
\|\partial z\|_{\mathbb{Z}} \leq(n-1)\|z\|_{\mathbb{Z}} .
$$

Proof. By Lemma 1.2, we may choose an integral cycle satisfying condition (1).

Let us suppose that the pseudomanifold $P$ associated to $z$ is disconnected. Then $P$ decomposes into a finite collection of connected pseudomanifolds $P_{1}, \ldots, P_{k}$ such that $c\left(P_{1}\right)+\ldots+c\left(P_{k}\right)=c(P)$. Each $P_{i}$ represents an integral cycle $z_{i}$, and if $d_{i} \in \mathbb{Z}$ is defined by the equation $\left[z_{i}\right]=d_{i}[M, \partial M]_{\mathbb{Z}}$, then $d_{1}+\ldots+d_{k}=d$. Therefore, there exists $i_{0} \in\{1, \ldots, k\}$ such that $d_{i_{0}} \neq 0$ and $c\left(P_{i_{0}}\right) /\left|d_{i_{0}}\right| \leq c(P) / d$. After replacing $z$ with $\pm z_{i_{0}}$, we may suppose that our cycle $z$ satisfies conditions (1) and (2).

As for condition (3), first note that since $P$ is now connected, if it has an $n$-simplex with $n+1$ boundary faces, then $P$ consists of a single simplex $\Delta^{n}$ with no identifications between its faces. In particular, $z$ consists of a single singular simplex $\sigma: \Delta^{n} \rightarrow M$ such that $\sigma\left(\partial \Delta^{n}\right) \subseteq \partial M$, so $\sigma$ has a welldefined degree, which cannot be null because $z$ is homologically non-trivial. In other words, there exists a nonzero degree map from the topological ball to $M$, and this implies that $\|M, \partial M\|=0$, against our assumptions. It remains 
to see that $P$ can be chosen not to have any simplex with $n$ boundary faces. Suppose that $P$ contains a simplex $\Delta_{1}^{n}$ having exactly one non-boundary face. Then $\Delta_{1}^{n}$ is adjacent to another simplex $\Delta_{2}^{n}$ of $P$. Roughly speaking, we remove from $z$ the singular simplex $\sigma_{1}$ corresponding to $\Delta_{1}^{n}$, and modify the singular simplex corresponding to $\Delta_{2}^{n}$ by suitably "expanding" it to compensate the removal of $\sigma_{1}$. More precisely, if $z=\sum_{i=1}^{k} \varepsilon_{i} \sigma_{i}$, then there exist a map $f:(|P|, \partial|P|) \rightarrow(M, \partial M)$ and singular simplices $\hat{\sigma}_{i}: \Delta^{n} \rightarrow|P|$ such that $\sigma_{i}=f \circ \hat{\sigma}_{i}$ for every $i=1, \ldots, k$. Let us denote by $F^{1} \subseteq \Delta_{1}^{n}$, $F^{2} \subseteq \Delta_{2}^{n}$ the pair of faces glued in $|P|$, and by $\varphi: F^{1} \rightarrow F^{2}$ their affine identification. Let $Q$ be the space obtained by gluing $\Delta_{1}^{n}$ and $\Delta_{2}^{n}$ along $\varphi$. We observe that the inclusion $\Delta_{1}^{n} \sqcup \Delta_{2}^{n} \hookrightarrow \Delta_{1}^{n} \sqcup \ldots \sqcup \Delta_{k}^{n}$ induces a welldefined map $\theta: Q \rightarrow|P|$, and we fix a homeomorphism $\psi: \Delta^{n} \rightarrow Q$ that restricts to the identity on $\partial \Delta_{2}^{n} \backslash F^{2}$. Finally, we define the singular simplex $\sigma_{2}^{\prime}: \Delta^{n} \rightarrow M$ by setting $\sigma_{2}^{\prime}=f \circ \theta \circ \psi$.

Let us set $z^{\prime}=\varepsilon_{2} \sigma_{2}^{\prime}+\sum_{i=3}^{k} \varepsilon_{i} \sigma_{i}$, and let $P^{\prime}$ be the pseudomanifold obtained by removing from $P$ the simplex $\Delta_{1}^{n}$ (and ignoring the only pairing involving a face of $\left.\Delta_{1}^{n}\right)$. It is readily seen that $z^{\prime}$ is still a relative cycle in $C_{n}(M, \partial M ; \mathbb{Z})$. What is more, we have $\left[z^{\prime}\right]=[z]=d \cdot[M, \partial M]_{\mathbb{Z}}$ in $H_{n}(M, \partial M ; \mathbb{Z})$. By construction, $z^{\prime}$ admits $P^{\prime}$ as associated pseudomanifold, $\left|P^{\prime}\right|$ is connected and $c\left(P^{\prime}\right)=c(P)-1$. As a consequence, the cycle $z^{\prime}$ satisfies $(1)$ and $(2)$, and $c\left(P^{\prime}\right)<c(P)$. If $z^{\prime}$ still has some simplex with exactly $n$ boundary faces of codimension one, then we may iterate our procedure until we get a cycle satisfying (1) and (2), and having no simplices with $n$ boundary faces. Since at every step the number of simplices of the associated pseudomanifold decreases, this iteration must come to an end.

Proof of Theorem 1. Let $M$ be a 3-manifold. We want to prove that

$$
\|M, \partial M\| \geq \frac{3}{4}\|\partial M\| \text {. }
$$

We may assume that $M$ is connected and oriented. If $\|\partial M\|=0$, there is nothing to prove, so we may assume that $\|\partial M\|>0$, whence also $\|M, \partial M\|>$ 0 .

Let $\varepsilon>0$ be given. We choose an integral cycle $z \in C_{3}(M, \partial M ; \mathbb{Z})$ with associated pseudomanifold $P$ that satisfies all the properties described in Proposition 2.1. Recall that $z_{P} \in C_{3}(|P|, \partial|P| ; \mathbb{Z})$ is the relative cycle represented by the (signed) sum of the simplices of $P$, and that $P$ comes with a map $f:(|P|, \partial|P|) \rightarrow(M, \partial M)$ such that $f_{*}\left(z_{P}\right)=z$.

For $i=0, \ldots, 4$, let us denote by $t_{i}$ the number of 3 -simplices of $P$ having exactly $i$ boundary 2 -faces. Our choice for $z$ implies that $t_{3}=t_{4}=0$, so Lemma 1.3 gives

$$
d \cdot\|\partial M\| \leq\|\partial z\|_{\mathbb{Z}}=c(\partial P)=t_{1}+2 t_{2} .
$$

Let $S_{1}, \ldots, S_{h}$ be the boundary components of $M$, and for every $i=1, \ldots, h$, let $Y_{i}^{1}, \ldots, Y_{i}^{k_{i}}$ be the connected components of $\partial|P|$ that are taken into $S_{i}$ by $f$. Since $P$ is orientable, each $Y_{i}^{j}$ is a closed orientable surface. Let $d_{i}^{j}$ 
be the degree of the map $\left.f\right|_{Y_{i}^{j}}: Y_{i}^{j} \rightarrow S_{i}$. Since $\left[f_{*}\left(\partial z_{P}\right)\right]=\left[\partial f_{*}\left(z_{P}\right)\right]=[\partial z]$ we have $\sum_{j=1}^{k_{i}} d_{i}^{j}=d$ for every $i=1, \ldots, h$, whence

$$
\sum_{j=1}^{k_{i}}\left\|Y_{i}^{j}\right\| \geq d \cdot\left\|S_{i}\right\|
$$

(see Proposition 1.4).

Let us consider the space $H$ obtained by removing from $|P|$ a closed tubular neighbourhood of edges and vertices. Of course, $H$ is an orientable handlebody, so in particular $\partial H$ is an orientable surface. If we denote by $Z_{1}, \ldots, Z_{r}$ the boundaries of regular neighbourhoods of the vertices of $|P| \backslash$ $\partial|P|$, then each $Z_{l}$ is an orientable surface, and $\partial H$ is obtained from the union of the $Y_{i}^{j}$ 's and the $Z_{l}$ 's by tubing. Putting together Corollary 1.6 and inequality (4), this implies that

$$
\|\partial H\| \geq \sum_{i=1}^{h} \sum_{j=1}^{k_{i}}\left\|Y_{i}^{j}\right\|+\sum_{l=1}^{r}\left\|Z_{l}\right\| \geq \sum_{i=1}^{h} d \cdot\left\|S_{i}\right\|=d \cdot\|\partial M\| .
$$

Let us denote by $\Gamma$ the graph dual to $P$. Since vertices and edges of $\Gamma$ correspond respectively to 3 -simplices and pairs of identified faces of $P$, we compute

$$
\chi(\Gamma)=\left(t_{0}+t_{1}+t_{2}\right)-\frac{4 t_{0}+3 t_{1}+2 t_{2}}{2}=\frac{-2 t_{0}-t_{1}}{2} .
$$

The handlebody $H$ retracts onto $\Gamma$. As a consequence, if $g$ is the genus of $H$ we obtain

$$
1-g=\chi(H)=\chi(\Gamma)=\frac{-2 t_{0}-t_{1}}{2} .
$$

As we are assuming that $\|\partial M\|>0$, Equation (5) implies that $g \geq 2$, and can be rewritten as

$$
4 g-4 \geq d \cdot\|\partial M\|
$$

Putting together Equations (6) and (7) we obtain

$$
4 t_{0}+2 t_{1} \geq d \cdot\|\partial M\| \text {. }
$$

Using inequalities (3) and (8) we get

$$
4\left(t_{0}+t_{1}+t_{2}\right)=2\left(t_{1}+2 t_{2}\right)+\left(4 t_{0}+2 t_{1}\right) \geq 3 d \cdot\|\partial M\|,
$$

whence

$$
\frac{3}{4}\|\partial M\| \leq \frac{t_{0}+t_{1}+t_{2}}{d}=\frac{c(P)}{d}=\frac{\|z\|_{\mathbb{Z}}}{d} \leq\|M, \partial M\|+\varepsilon .
$$

Since $\varepsilon$ is arbitrary, this concludes the proof of Theorem 1 
Proof of Theorem 2, If $M$ is a 3-manifold with nonempty boundary, we say that $N$ is obtained from $M$ by adding a 1-handle if $N=M \cup_{f} H$, where $H=D^{2} \times[0,1]$ is a solid cylinder and $f: D^{2} \times\{0,1\} \rightarrow \partial M$ is a homeomorphism onto the image.

Proposition 2.2. Let $M$ be any 3-manifold with nonempty boundary, and let $N$ be obtained from $M$ by adding a 1-handle. Then

$$
\sigma(N) \leq \sigma(M)+3, \quad \sigma_{\infty}(N) \leq \sigma_{\infty}(M)+3 .
$$

Proof. Let $T$ be a copy of the standard 2-simplex, and fix an identification of the added 1-handle with the prism $T \times[0,1]$. For $i=0,1$, we denote by $\partial_{i} M$ the boundary component of $\partial M$ glued to $T \times\{i\}$. Observe that we may have $\partial_{0} M=\partial_{1} M$. The prism $T \times[0,1]$ can be triangulated by 3 simplices, in such a way that $T \times\{0\}$ and $T \times\{1\}$ appear as boundary faces of the triangulation. Let $\mathcal{T}$ be a minimal triangulation of $M$. Every component of $\partial M$ inherits from $\mathcal{T}$ a triangulation with at least two triangles, so we may choose distinct boundary faces $T_{0}, T_{1}$ of $\mathcal{T}$ and affine identifications $\varphi_{i}: T \times\{i\} \rightarrow T_{i}$ in such a way that $N$ is homeomorphic to the space obtained by gluing $M$ and $T \times[0,1]$ along the $\varphi_{i}$ 's. This space is endowed with a triangulation with $\sigma(M)+3$ tetrahedra, and this proves that

$$
\sigma(N) \leq \sigma(M)+3
$$

As for the stable $\Delta$-complexity, if $f: \widehat{M} \rightarrow M$ is any covering of degree $d$, then it is immediate that $f$ extends to a covering $\bar{f}: \widehat{N} \rightarrow N$ of degree $d$, where $\widehat{N}$ is obtained from $\widehat{M}$ by adding $d$ 1-handles. We thus get

$$
\frac{\sigma(\widehat{N})}{d} \leq \frac{\sigma(\widehat{M})+3 d}{d}=\frac{\sigma(\widehat{M})}{d}+3
$$

Since the covering $f: \widehat{M} \rightarrow M$ was arbitrary, this implies that

$$
\sigma_{\infty}(N) \leq \sigma_{\infty}(M)+3
$$

and concludes the proof of Proposition 2.2.

We can now easily conclude the proof of Theorem 2, In fact, if $M$ is a Seifert manifold with nonempty boundary then we know from the introduction that $\sigma_{\infty}(M)=0$. Therefore, if $N$ is obtained by consecutively adding $h$ handles on $M$, Proposition 2.2 implies that

$$
\sigma_{\infty}(N) \leq \sigma_{\infty}(M)+3 h=3 h
$$

On the other hand, every boundary component of $M$ has null Euler characteristic, and no boundary component of any manifold obtained by adding 1-handles to $M$ has positive Euler characteristic, so Proposition 1.5 implies that $\|\partial N\|=4 h$. Putting together this inequality with Inequality (10), and recalling that stable $\Delta$-complexity always bounds the simplicial volume from above, we get

$$
\|N, \partial N\| \leq \sigma_{\infty}(N) \leq \frac{3}{4}\|\partial N\|
$$


Finally, Theorem 1 implies that all the inequalities in (11) are in fact equalities, which finishes the proof of Theorem 2.

\section{THE CASE OF BOUNDARY IRREDUCIBLE ASPHERICAL MANIFOLDS}

The estimate provided by Theorem 1 may be improved in the case of boundary irreducible aspherical 3-manifolds. This additional hypothesis can be exploited to construct a topological straightening for simplices. The existence of such an operator implies in turn that the simplicial volume may be computed just by looking at straight chains, that verify interesting additional topological properties.

A topological straightening. The straightening procedure for simplices was introduced by Thurston in Thu79, in order to bound from below the simplicial volume of hyperbolic manifolds. If $M$ admits a nonpositively curved Riemannian metric, then one may associate to every singular simplex $\sigma$ in $M$ a straight $\operatorname{simplex} \operatorname{str}(\sigma)$, which is uniquely determined by the vertices of $\sigma$ and the homotopy classes (relative to the endpoints) of the edges of $\sigma$. We now extend this construction to the case we are interested in.

Until the end of the section, we denote by $M$ a boundary irreducible aspherical manifold such that $\partial M$ is also aspherical. Let $p: \widetilde{M} \rightarrow M$ be the universal covering of $M$, observe that $\widetilde{M}$ is contractible, and fix an identification of $\pi_{1}(M)$ with the group $\Gamma$ of the covering automorphisms of $p: \widetilde{M} \rightarrow M$. Since $M$ is boundary irreducible, the restriction of $p$ to any connected component of $\partial \widetilde{M}$ is a universal covering of a component of $\partial M$. Moreover, since $\partial M$ is aspherical, every component of $\partial \widetilde{M}$ is contractible. Using these facts and proceeding inductively on the dimension of simplices, one can prove the following:

Proposition 3.1. For $R=\mathbb{Z}, \mathbb{Q}, \mathbb{R}$, there exists a $\Gamma$-equivariant chain map

$$
\widetilde{\operatorname{str}}_{*}: C_{*}(\widetilde{M} ; R) \rightarrow C_{*}(\widetilde{M} ; R)
$$

which satisfies the following properties:

(1) For every simplex $\sigma \in S_{i}(\widetilde{M})$, the chain $\widetilde{\operatorname{str}}_{i}(\sigma)$ consists of a single simplex. Moreover, $\sigma$ and $\widetilde{\operatorname{str}}_{i}(\sigma)$ share the same vertices.

(2) If two simplices $\sigma, \sigma^{\prime} \in S_{i}(\widetilde{M})$ share the same vertices, then $\widetilde{\operatorname{str}}_{i}(\sigma)=$ $\widetilde{\operatorname{str}}_{i}\left(\sigma^{\prime}\right)$.

(3) If $\sigma \in S_{i}(\partial \widetilde{M})$, then $\widetilde{\operatorname{str}}_{i}(\sigma) \in S_{i}(\partial \widetilde{M})$, so $\widetilde{\operatorname{str}_{*}}$ induces a $\Gamma$-equivariant chain map $C_{*}(\widetilde{M}, \partial \widetilde{M} ; R) \rightarrow C_{*}(\widetilde{M}, \partial \widetilde{M} ; R)$, which will still be denoted by $\widetilde{\operatorname{str}}_{*}$.

(4) The map $\widetilde{\operatorname{str}_{*}}: C_{*}(\widetilde{M}, \partial \widetilde{M} ; R) \rightarrow C_{*}(\widetilde{M}, \partial \widetilde{M} ; R)$ is $\Gamma$-equivariantly homotopic to the identity. 
By the previous proposition, $\widetilde{\operatorname{str}}_{*}$ induces a map $\operatorname{str}_{*}: S_{*}(M) \rightarrow S_{*}(M)$ which induces in turn a chain map $\operatorname{str}_{*}: C_{*}(M, \partial M ; R) \rightarrow C_{*}(M, \partial M ; R)$. Simplices that lie in the image of $\operatorname{str}_{*}$ will be called straight.

Lemma 3.2. Let $\sigma$ be a straight $k$-simplex, $k \geq 2$, with image in $M$, and suppose that $\sigma$ is not supported on $\partial M$. Then:

(1) at most one $(k-1)$-face of $\sigma$ lies on $\partial M$;

(2) if $k=3$ and no 2-face of $\sigma$ lies on $\partial M$, then at most two edges of $\sigma$ lie on $\partial M$;

(3) if $k=3$ then there exist at most three edges of $\sigma$ in $\partial M$.

Proof. Let $\widetilde{\sigma}$ be a fixed lift of $\sigma$ to $\widetilde{M}$. If there exists a component of $\partial \widetilde{M}$ containing $m+1$ vertices of $\widetilde{\sigma}$, then there exists a simplex $\widetilde{\sigma}^{\prime} \in S_{k}(\widetilde{M})$ having the same vertices of $\widetilde{\sigma}$ and an $m$-dimensional face supported on $\partial \widetilde{M}$. By Proposition 3.1, this implies that $\widetilde{\sigma}=\widetilde{\operatorname{str}}_{k}\left(\widetilde{\sigma}^{\prime}\right)$ also has an $m$-dimensional face supported in $\partial \widetilde{M}$. In particular, the assumption that $\sigma$ is not supported on $\partial M$ implies that no component of $\partial \widetilde{M}$ contains all the vertices of $\widetilde{\sigma}$.

(1) If $\sigma$ has two faces on $\partial M$, then the vertices of $\widetilde{\sigma}$ are contained in the same connected component of $\partial \widetilde{M}$, a contradiction.

(2) Suppose that $\sigma$ has at least three edges on $\partial M$. Since $k=3$, the union of the corresponding edges of $\widetilde{\sigma}$ is connected, so at least three vertices of $\widetilde{\sigma}$ lie on the same connected component of $\partial \widetilde{M}$, and at least one 2-face of $\sigma$ is supported on $\partial M$.

(3) If four edges of $\sigma$ lie on $\partial M$, then as in (2), the union of the corresponding edges of $\widetilde{\sigma}$ is connected. But the vertices of these four edges of the 3 -simplex are all the vertices of the 3 -simplex, which all lie on the same connected component of $\partial \widetilde{M}$, a contradiction.

The fact that $M$ supports a relative straightening can be used to improve Proposition 2.1 as follows:

Proposition 3.3. Suppose that $M$ is an aspherical and boundary irreducible $n$-manifold, $n \geq 2$, and that $\partial M$ is also aspherical. Let $\varepsilon>0$ be fixed and assume that $\|M, \partial M\|>0$. Then, there exists a relative integral cycle $z \in C_{n}(M, \partial M ; \mathbb{Z})$ with associated pseudomanifold $P$ such that the following conditions hold:

(1) $[z]=d \cdot[M, \partial M]_{\mathbb{Z}}$ in $H_{n}(M, \partial M ; \mathbb{Z})$ for some integer $d>0$, and

$$
\frac{\|z\|_{\mathbb{Z}}}{d} \leq\|M, \partial M\|+\varepsilon ;
$$

(2) every simplex of $P$ has at most one $(n-1)$-dimensional boundary face;

(3) if $n=3$, then every simplex of $P$ without 2-dimensional boundary faces has at most two edges contained in $\partial|P|$ and every simplex has at most three edges in $\partial|P|$. 
Proof. Let $z^{\prime}$ be an integral cycle satisfying conditions (1) and (2) of Proposition 2.1, and set $z=\operatorname{str}_{n}\left(z^{\prime}\right) \in C_{n}(M, \partial M ; \mathbb{Z})$. As usual, we understand that no simplex appearing in $z$ is supported in $\partial M$ (otherwise, we may just remove it from $z$ without modifying the class of $z$ in $C_{n}(M, \partial M ; \mathbb{Z})$ and decreasing $\|z\|_{\mathbb{Z}}$ ). Point (1) descends from the fact that the straightening operator is norm nonincreasing and homotopic to the identity, while points (2) and (3) follow from Lemma 3.2 .

Proof of Theorem 4. Let us suppose that $M$ is an aspherical boundary irreducible 3-manifold. As usual, we may also suppose that $M$ is oriented, and that $\|M, \partial M\|>0$ (otherwise there is nothing to prove). In order to exploit the machinery introduced above, we first reduce to the case when $\partial M$ is also aspherical. So, let us suppose that a component $S$ of $\partial M$ is a sphere. Since $M$ is aspherical, $S$ is homotopically trivial, whence homologically trivial, in $M$. This implies that $\partial M=S$, so $\|\partial M\|=0$ and the conclusion of Theorem 4 is trivially satisfied. (In fact, using the Poincaré conjecture one can prove that the only aspherical 3-manifold with at least one spherical boundary component is the ball.) Therefore, henceforth we suppose that $\partial M$ is also aspherical.

We denote by $z$ the cycle provided by Proposition 3.3 , and by $P$ the associated pseudomanifold. As usual, let $z_{P} \in C_{3}(|P|, \partial|P| ; \mathbb{Z})$ be the relative cycle represented by the (signed) sum of the simplices of $P$, and let $f:(|P|, \partial|P|) \rightarrow(M, \partial M)$ be such that $f_{*}\left(z_{P}\right)=z$. Also recall that the space $\partial|P|$ is an orientable surface. The equality $f_{*}\left(z_{P}\right)=d \cdot[M, \partial M]_{\mathbb{Z}}$ implies that

$$
\left(\left.f\right|_{\partial|P|}\right)_{*}([\partial P])=d \cdot[\partial M],
$$

where $[\partial P]$ (resp. $[\partial M]$ ) is the sum of the real fundamental classes of the components of $\partial|P|$ (resp. of $\partial M)$.

Let $\Omega_{i}$, for $i=0, \ldots, 4$, be the set of simplices of $P$ having exactly $i$ boundary 2-faces. As usual, we denote by $t_{i}$ the number of elements of $\Omega_{i}$. By Proposition 3.3 we have $\Omega_{2}=\Omega_{3}=\Omega_{4}=\emptyset$, so

$$
t_{2}=t_{3}=t_{4}=0, \quad\|z\|_{\mathbb{Z}}=t_{0}+t_{1} .
$$

Since $\partial|P|$ admits a triangulation with $t_{1}$ triangles we have $t_{1} \geq\|\partial|P|\|$. Also, Equation (12) implies that $\|\partial|P|\| \geq d\|\partial M\|$, so

$$
t_{1} \geq d \cdot\|\partial M\| \text {. }
$$

In order to prove Theorem 4 we now need to bound $t_{0}$ from below. Let us first introduce some definitions. We say that an edge $e$ of the 2-dimensional pseudomanifold $\partial P$ is nice if $e$ is the edge of a simplex in $\Omega_{0}$. We also say that an edge of $\partial P$ is bad if it is not nice. An edge of the topological realization $\partial|P|$ is nice if it is the image of at least one nice edge in $\partial P$. An edge in $\partial|P|$ is bad if it is not nice. Notice that bad edges in $\partial|P|$ are not the image of bad edges in $\partial P$, since a nice edge in $\partial|P|$ will be the image of a certain number of nice edges in $\partial P$ and necessarily two bad edges of $\partial P$ 
corresponding to the two (possibly nondistinct) tetrahedra having as 2-faces the 2 -faces in $\partial|P|$ containing the original nice edge.

Lemma 3.4. Let e be a bad edge of $\partial|P|$, let $T_{j}, T_{j^{\prime}}$ be the triangles of $\partial P$ adjacent to $e$, and let $\Delta_{j}^{3}$ (resp. $\left.\Delta_{j^{\prime}}^{3}\right)$ be the simplex of $P$ containing $T_{j}$ (resp. $\left.T_{j^{\prime}}\right)$. If $F_{j}$ (resp. $\left.F_{j^{\prime}}\right)$ is the 2 -face of $\Delta_{j}^{3}$ (resp. $\Delta_{j^{\prime}}^{3}$ ) such that $e=F_{j} \cap T_{j}=F_{j^{\prime}} \cap T_{j^{\prime}}$, then $F_{j}, F_{j^{\prime}}$ are glued to each other in $|P|$.

Proof. Let $\overline{\Delta^{3}}$ be the simplex of $P$ glued to $\Delta_{j}^{3}$ along $F_{j}$. Denote by $\bar{F}$ the face of $\overline{\Delta^{3}}$ paired to $F_{j}$. We consider separately the following cases.

(1) Suppose that $\overline{\Delta^{3}} \neq \Delta_{j}^{3}$ and $\overline{\Delta^{3}} \neq \Delta_{j^{\prime}}^{3}$. Since $e$ is bad, at least one face $F$ of $\overline{\Delta^{3}}$ has to lie on $\partial|P|$. Moreover, the conditions $\overline{\Delta^{3}} \neq \Delta_{j}^{3}, \overline{\Delta^{3}} \neq \Delta_{j^{\prime}}^{3}$ imply that $F$ cannot contain $e$, because otherwise $e$ would be adjacent to 3 boundary faces of $P$ (counted with multiplicities). Therefore, $\overline{\Delta^{3}}$ contains four edges which lie on $\partial|P|$, and this contradicts point (4) of Proposition 3.3 .

(2) Suppose that $\overline{\Delta^{3}}=\Delta_{j}^{3}$. Since $\Delta_{j}^{3}$ has at most three edges on $\partial|P|$, the unique boundary edge of $F_{j}$ is paired to the unique boundary edge of $\bar{F}$. As a consequence we have $\bar{F}=F_{j^{\prime}}$, as desired (note that in this case we have $T_{j}=T_{j^{\prime}}$ and $\Delta_{j}^{3}=\Delta_{j^{\prime}}^{3}$.

(3) Suppose that $\overline{\Delta^{3}}=\Delta_{j^{\prime}}^{3}$. Using that $\Delta_{j^{\prime}}^{3}$ has at most three edges on $\partial|P|$, one may easily show that $\bar{F}=F_{j^{\prime}}$, whence the conclusion.

We denote by $\Gamma \subseteq \partial|P|$ the union of all the nice edges of $\partial P$. Then, $\Gamma$ is a (possibly disconnected) graph in $\partial|P|$.

Let $\Gamma_{i}$, for $i=1, \ldots, s$ be the connected components of $\Gamma$. For each $i$ we denote by $N_{i}=N\left(\Gamma_{i}\right)$ a closed regular neighbourhood of $\Gamma_{i}$ in $\partial|P|$, chosen in such a way that $N_{i} \cap T$ is a regular neighbourhood of $\Gamma_{i} \cap T$ for every triangle $T$ of $\partial|P|$ and $N_{i} \cap N_{j}=\emptyset$ whenever $i \neq j$. We also set $N=\cup_{i=1}^{s} N_{i}$ and $W=\overline{\partial|P| \backslash N}$. Finally, we denote by $W_{1}, \ldots, W_{r}$ the components of $W$ (see Figure 1).

We will prove that $\left.f\right|_{\partial|P|}$ is homotopic to a map which is constant on each $W_{i}$. Since $\left.f\right|_{\partial|P|}$ has degree $d$, this will imply that $N$ has to be sufficiently complicated, and this will prove in turn that the number of nice edges of $\partial|P|$ cannot be too small.

Lemma 3.5. Let $\gamma \subseteq W$ be a loop. Then $f(\gamma)$ is null-homotopic in $\partial M$.

Proof. Let $W_{i}$ be the component of $W$ containing $\gamma$. Let $\left\{T_{j}\right\}_{j \in J}$ be the family of triangles of $\partial P$ that intersect $W_{i}$, and let $\Delta_{j}^{3}$ be the simplex in $P$ containing $T_{j}$. We denote by $v_{j}$ the vertex of $\Delta_{j}^{3}$ opposite to $T_{j}$, and set $\widehat{T}_{j}=T_{j} \cap W=T_{j} \cap W_{i}$. Observe that by definition we have $W_{i}=\cup_{j \in J} \widehat{T}_{j}$. Since each edge that intersects $W_{i}$ is bad, Lemma 3.4 implies that all the vertices $\left\{v_{j}\right\}_{j \in J}$ coincide in $|P|$. This implies in turn that the inclusion $W_{i} \hookrightarrow \partial|P|$ extends to an inclusion $C\left(W_{i}\right) \hookrightarrow|P|$ of the topological cone over $W_{i}$. Since $C\left(W_{i}\right)$ is contractible, it follows that $\gamma$ is null-homotopic 

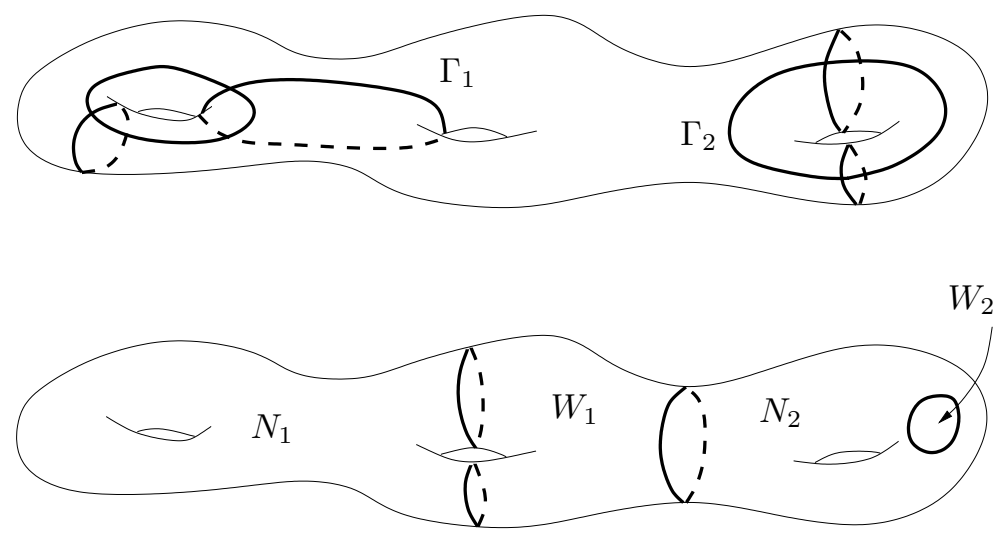

Figure 1. $N_{i}$ is a regular neighbourhood of $\Gamma_{i}$ on $\partial|P|$ for $i=1,2$.

in $|P|$. Therefore, $f(\gamma)$ is null-homotopic in $M$, whence in $\partial M$ due to the boundary irreducibility of $M$.

Corollary 3.6. There exists a map $g: \partial|P| \rightarrow \partial M$ homotopic to $\left.f\right|_{\partial|P|}$ and such that $\left.g\right|_{W_{i}}$ is constant for every $i=1, \ldots, r$.

Proof. Each component $W_{i}$ of $W$ is a compact orientable surface. Since $\partial M$ is aspherical, by Lemma 3.5 the map $\left.f\right|_{W_{i}}$ can be homotoped to a constant map $g_{i}: W_{i} \rightarrow \partial M$ via a homotopy $H_{i}: W_{i} \times[0,1] \rightarrow \partial M$ such that $H_{i}(x, 0)=g_{i}(x)$ and $H_{i}(x, 1)=f(x)$ for every $x \in W_{i}$. We now need to define a global map $g: \partial|P| \rightarrow \partial M$ such that $\left.g\right|_{W_{i}}=g_{i}$ for every $i$.

We piecewise define $g$ as follows. For every component $\gamma$ of $\partial W$ we denote by $N_{\gamma}$ the component of $N$ containing $\gamma$. We also fix a collar $C(\gamma) \cong \gamma \times[0,1]$ of $\gamma$ in $N_{\gamma}$, in such a way that $\gamma$ is identified with $\gamma \times\{0\} \subseteq C(\gamma)$ and all the chosen collars are disjoint, and we set

$$
N^{\prime}=\overline{N \backslash \bigcup_{\gamma \subseteq \partial W} C(\gamma)} .
$$

Of course, $N^{\prime}$ is homeomorphic to $N$. More precisely, there exists a homeomorphism $t: N \rightarrow N^{\prime}$ such that the composition $N \rightarrow N^{\prime} \hookrightarrow N$ is homotopic to the identity of $N$, and $t(x, 0)=(x, 1)$ for every $x \in \gamma \subseteq C(\gamma) \cong \gamma \times[0,1]$, where $\gamma$ is any component of $\partial N=\partial W$. We set $\left.g\right|_{N^{\prime}}=\left.f\right|_{N} \circ t^{-1}$. It remains to properly define $g$ on the annuli $C(\gamma)$. To this aim, if $\gamma$ is any component of $\partial W_{i}$ and $(x, s) \in \gamma \times[0,1] \cong C(\gamma)$, then we set $g(x, s)=H_{i}(x, s)$. It is easy to check that the resulting map $g$ is well-defined, continuous and homotopic to $f$.

The following proposition provides the key step in the proof of Theorem 4 . We denote by $E_{\text {nice }}$ the number of nice edges of $\partial|P|$. 


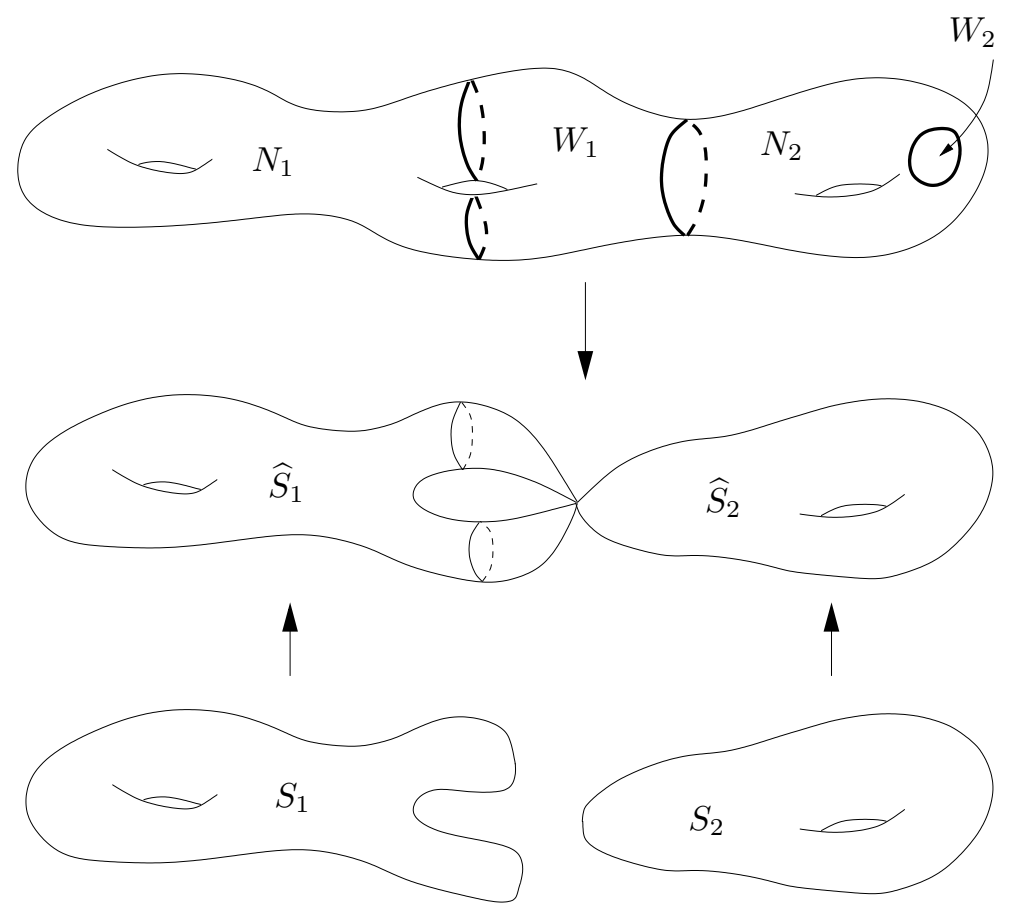

Figure 2. The construction described in Proposition 3.7

\section{Proposition 3.7. We have}

$2 \cdot E_{\text {nice }} \geq 4 \cdot \sharp\{$ connected components of $\partial M\}+d \cdot\|\partial M\|$.

Proof. For every $i=1, \ldots, s$, let $S_{i}$ be the closed orientable surface obtained from $N_{i}$ by collapsing to a point each connected component of $\partial N_{i}$ (we understand that distinct components of $\partial N_{i}$ give rise to distinct points). If $e_{i}$ is the number of nice edges of $\Gamma_{i}$, then

$$
\chi\left(S_{i}\right) \geq 1+\chi\left(N_{i}\right)=1+\chi\left(\Gamma_{i}\right) \geq 2-e_{i} .
$$

Summing over $i$, we obtain

$$
E_{\text {nice }} \geq 2 \cdot s-\sum_{i=1}^{s} \chi\left(S_{i}\right) .
$$

Since for closed oriented surfaces $S$ the simplicial volume is equal to $\|S\|=$ $-2 \chi(S)$ unless $S$ is homeomorphic to the 2 -sphere in which case $\left\|S^{2}\right\|=$ $2-\chi\left(S^{2}\right)$, the latter inequality can be rewritten as

$$
2 \cdot E_{\text {nice }} \geq 4 \cdot \sharp\left\{i \mid S_{i} \nsucceq S^{2}\right\}+\sum_{i=1}^{s}\left\|S_{i}\right\| .
$$

It remains to show that

$$
\sharp\left\{i \mid S_{i} \supsetneqq S^{2}\right\} \geq \sharp\{\text { connected components of } \partial M\}
$$


and

$$
\sum_{i=1}^{s}\left\|S_{i}\right\| \geq d \cdot\|\partial M\|
$$

Let $\widehat{S}$ be the space obtained from $\partial|P|$ by collapsing to a point each connected component of $W$ (again, we understand that distinct components of $W$ give rise to distinct points), and let us denote by $\pi: \partial|P| \rightarrow \widehat{S}$ the quotient map (see Figure 2). We observe that $S_{i}$ canonically projects onto $\widehat{S}$ and we denote by $p: \cup_{i=1}^{s} S_{i} \rightarrow \widehat{S}$ the resulting map.

Let us consider the map $g: \partial|P| \rightarrow \partial M$ provided by Corollary 3.6. Being constant on the components of $W$, the map $g$ induces a map $\widehat{g}$ on $\widehat{S}$ and by precomposition by $p$ a map $\widehat{g}_{S}$ on $\cup_{i=1}^{s} S_{i}$ such that the following diagram commutes:

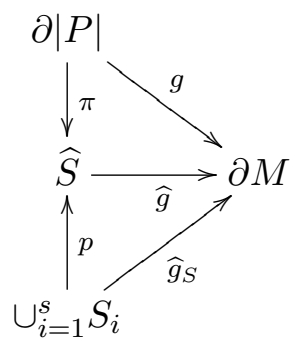

The orientation on $\partial|P|$ induces an orientation on each $N_{i}$, whence on each $S_{i}$, endowing $\cup_{i=1}^{s} S_{i}$ and also $\widehat{S}=p\left(\cup_{i=1}^{s} S_{i}\right)$ with a fundamental class. Since $g$ is homotopic to $\left.f\right|_{\partial|P|}$, it follows from the commutativity of the previous diagram and the fact that $\pi_{*}([\partial|P|])=[\widehat{S}]=p_{*}\left(\left[\cup_{i=1}^{s} S_{i}\right]\right)$ that

$$
\left(\widehat{g}_{S}\right)_{*}\left(\left[\cup_{i=1}^{s} S_{i}\right]\right)=\widehat{g}_{*}([\widehat{S}])=g_{*}([\partial|P|])=d \cdot[\partial M],
$$

where the last equality is a consequence of Equation (12). Thus, for every connected component $M_{0}$ of $\partial M$ there exists at least one $S_{i}$ not homeomorphic to $S^{2}$ and mapped to $M_{0}$ by $\widehat{g}_{S}$, proving the first desired inequality. Finally, since $\left(\widehat{g}_{S}\right)_{*}$ is norm nonincreasing, we obtain from (14) the second desired inequality

$$
d \cdot\|\partial M\|=\left\|\left(\widehat{g}_{S}\right)_{*}\left(\left[\cup_{i=1}^{s} S_{i}\right]\right)\right\| \leq \sum_{i=1}^{s}\left\|S_{i}\right\|,
$$

which finishes the proof of the proposition.

To conclude the proof of Theorem 4 note that by definition every nice edge of $\partial|P|$ is contained in at least one simplex in $\Omega_{0}$. Moreover, by point (4) of Proposition 3.3 every simplex in $\Omega_{0}$ has at most two edges on $\partial|P|$, so the inequality $t_{0} \geq E_{\text {nice }} / 2$ holds. Putting this inequality together with Proposition 3.7 and Inequality 13 we get

$$
d(\|M, \partial M\|+\varepsilon) \geq\|z\|=t_{0}+t_{1} \geq d \cdot \frac{\|\partial M\|}{4}+d \cdot\|\partial M\|=\frac{5 d}{4}\|\partial M\|,
$$

which proves the theorem since $\varepsilon$ is arbitrary. 
Proof of Theorem 5. We show that the $\Delta$-complexity of the product $M_{g}=S_{g} \times[0,1]$ of a surface of genus $g \geq 1$ with an interval is equal to

$$
\sigma\left(M_{g}\right)=10 \cdot(g-1)+6 .
$$

For the inequality $\sigma\left(M_{g}\right) \leq 10 \cdot(g-1)+6$ we exhibit a topological triangulation of $M_{g}$ with the prescribed amount of top dimensional simplices. Let us realize $S_{g}$ as the space obtained by gluing the sides of a $4 g$-gon, as described in Figure 3-left, corresponding to the presentation

$$
\left.\left.\left\langle x_{1}, \ldots, x_{2 g}\right| x_{1} \cdot \ldots \cdot x_{2 g} \cdot\left(x_{1}\right)^{-1} \cdot \ldots \cdot\left(x_{2 g}\right)^{-1}\right)=1\right\rangle
$$

of $\pi_{1}\left(\Sigma_{g}\right)$ (it is easy to check the cellular structure induced on the quotient has exactly one vertex, so it must be homeomorphic to $\Sigma_{g}$ by an obvious Euler characteristic argument).

We decompose the polygon in $2 g-2$ squares and 2 triangles as indicated in Figure 3 -right. We orient each of the edges of that decomposition so that the orientations match on edges being identified in $\Sigma_{g}$. In the product $\Sigma_{g} \times[0,1]$ a square will be triangulated in 5 simplices so that for every oriented edge with vertices $x, y$, the product with $[0,1]$ is triangulated in two simplices by an edge from $\{(x, 0)\}$ to $\{(y, 1)\}$ if the orientation of the edge goes from $x$ to $y$ (see Figure 4 ). As for the two products of triangles with $[0,1]$, they can each be triangulated in 3 simplices respecting the imposed triangulation of their boundaries (see Figure 5). We have thus constructed a triangulation of $M_{g}=S_{g} \times[0,1]$ in $(2 g-2) \cdot 5+2 \cdot 3$ tetrahedra.
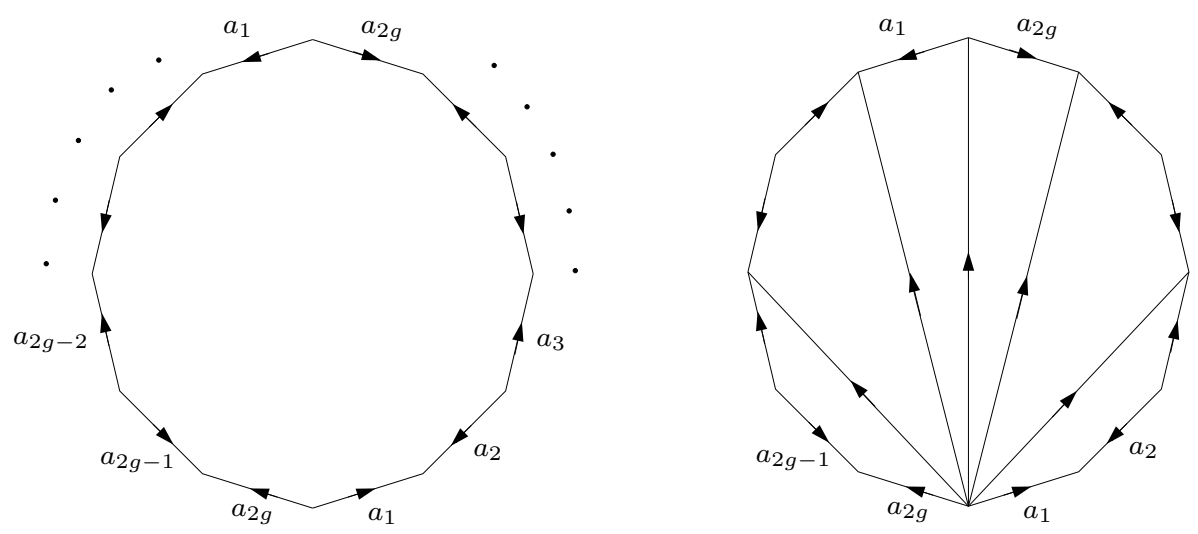

Figure 3. The decomposition of $S_{g}$ into $2 g-2$ squares and 2 triangles, in the case $g=3$.

It remains to prove the other inequality $\sigma\left(M_{g}\right) \geq 10 \cdot(g-1)+6$. Let $T$ be a triangulation of $M_{g}$ with simplices $\Delta_{1}^{3}, \ldots, \Delta_{N}^{3}$. We need to show that $N \geq 10(g-1)+6$.

We start by choosing a straightening operator on $\left(M_{g}, \partial M_{g}\right)$ with additional symmetry. To do so, we endow $\Sigma_{g}$ with a hyperbolic structure and 

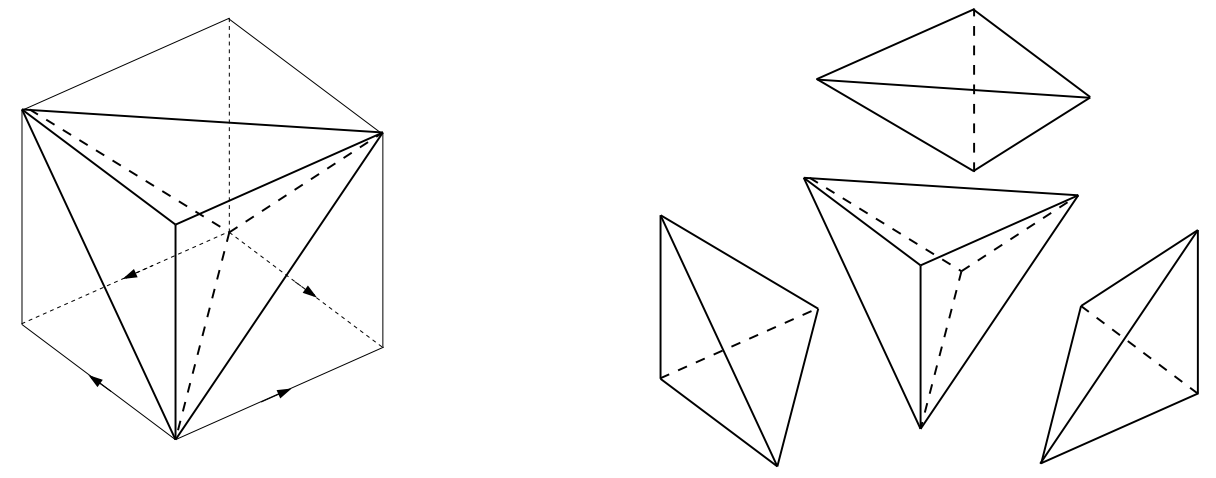

Figure 4. Any orientation of the edges of a square $S$ with one source and one sink (on the left) determines a decomposition of the cube $S \times[0,1]$ into 5 simplices (on the right).
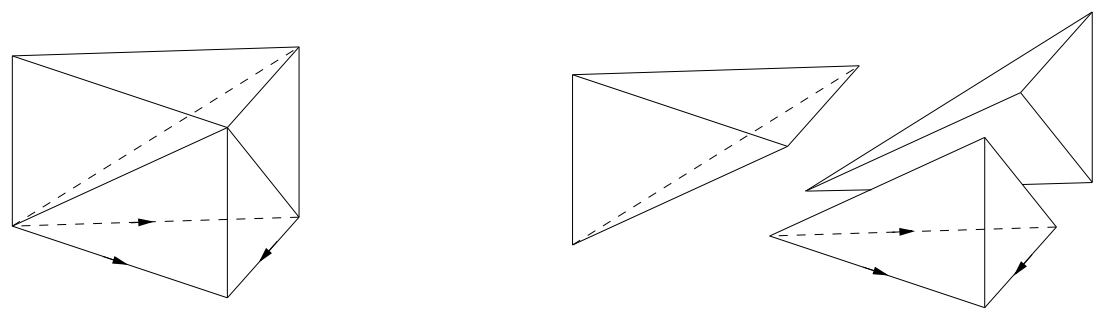

Figure 5. A triangle $T$ with oriented edges determines a decomposition of the prysm $T \times[0,1]$ into 3 tetrahedra.

consider on $\Sigma_{g}$ the barycentric straightening, associating to any singular simplex $\sigma: \Delta^{q} \rightarrow \Sigma_{g}$, for $q \geq 0$, a straightened $\operatorname{simplex} \operatorname{str}_{\text {bar }}(\sigma)$ (see Rat94, Chapter 11] for details). Note that the barycentric straightening has the property that it does not depend, in constant curvature, on the order of the vertices of $\sigma$ (in contrast to the straightening obtained by geodesic coning). Consider the resulting product straightening on $\Sigma_{g} \times[0,1]$, denoted by str, where on the interval $[0,1]$ we consider the affine straightening. Note that str still has the property that it does not depend on the order of the vertices.

For every $i=1, \ldots, N$, we fix an orientation-preserving parameterization $\sigma_{i}: \Delta^{3} \rightarrow \Delta_{i}^{3}$, and we say that a simplex of $T$ is inessential if the image of $\operatorname{str}\left(\sigma_{i}\right)$ lies on $\partial M_{g}$, and essential otherwise. We order the simplices of $T$ so that $\Delta_{1}^{3}, \ldots, \Delta_{N_{0}}^{3}$ are essential, and $\Delta_{N_{0}+1}^{3}, \ldots, \Delta_{N}^{3}$ are not. We now define an orientable pseudomanifold $P$ as follows. The simplices of $P$ bijectively correspond to the essential simplices of $T$, and gluings in $P$ correspond to gluings between essential simplices of $T$. (This does not mean that $P$ is identified with a subset of $M_{g}$, since, for example, two essential simplices of 
$T$ may share an edge because they are glued to the same inessential simplex, so they may intersect in $M_{g}$, while being disjoint in $P$.)

We define a map $\operatorname{str}_{T}:|P| \rightarrow M_{g}$ which corresponds to the simultaneous straightening of all the essential simplices of $T$. To define the map $\operatorname{str}_{T}$ on $|P|$, we choose, for every $p \in|P|$, an $i \in\left\{1, \ldots, N_{0}\right\}$ such that $p \in\left|\Delta_{i}^{3}\right| \subseteq|P|$, and we set $\operatorname{str}_{T}(p)=\operatorname{str}\left(\sigma_{i}\right)(q)$, where $q \in \Delta_{i}^{3} \cong \Delta^{3}$ is any point which gets identified with $p$ under the natural map $\Delta_{i}^{3} \rightarrow\left|\Delta_{i}^{3}\right| \subseteq|P|$. Of course, if the point $p$ belongs to the 2-skeleton of $|P|$, then there may be several choices of $i$ and possibly also for $q \in \Delta^{3}$ (recall that distinct faces of $\Delta_{i}^{3}$ may be identified in $|P|$ ). However, since our straightening does not depend on the order of the vertices, one may easily check that $\operatorname{str}_{T}$ is indeed well-defined and continuous. Let us further see that $\operatorname{str}_{T}$ is a map of pairs

$$
\operatorname{str}_{T}:(|P|, \partial|P|) \rightarrow\left(M_{g}, \partial M_{g}\right) .
$$

If $F$ is any boundary face of $|P|$, then either $F$ corresponds to a boundary face of $T$, or it corresponds to a face of $T$ which is glued to an inessential simplex of $T$. In the first case we deduce that $\operatorname{str}_{T}(F) \subseteq \partial M_{g}$ from the fact that straightening preserves the space of singular simplices supported on $\partial M_{g}$. In the second case it is sufficient to observe that after straightening faces of inessential simplices are supported on $\partial M_{g}$.

Let us analyze the action of $\operatorname{str}_{T}$ on fundamental cycles. We first point out that, in general, we cannot assume that the sum $\sum_{i=1}^{N} \sigma_{i}$ is a relative cycle in $C_{n}\left(M_{g}, \partial M_{g} ; \mathbb{Z}\right)$. In fact, if two 2 -faces of $T$ are identified in $M_{g}$, then it may happen that the corresponding faces of the $\sigma_{i}$ 's, when considered as singular 2-simplices, differ by the precomposition with a nontrivial affine automorphism of the standard 2-simplex, and do not cancel each other in the algebraic boundary of $\sum_{i=1}^{N} \sigma_{i}$. This problem can be fixed by alternating each $\sigma_{i}$ as follows. For any singular 3 -simplex $\sigma$ we define the chain

$$
\operatorname{alt}(\sigma)=\frac{1}{(4) !} \sum_{\tau \in \mathfrak{S}_{4}}(-1)^{\operatorname{sgn}(\tau)} \sigma \circ \bar{\tau}
$$

where $\bar{\tau}$ is the unique affine diffeomorphism of the standard 3 -simplex $\Delta^{3}$ corresponding to the permutation $\tau$ of the vertices of $\Delta^{3}$. Now it is immediate that the real chain $z_{M}^{\mathbb{R}}=\operatorname{alt}\left(\sigma_{1}\right)+\ldots+\operatorname{alt}\left(\sigma_{N}\right)$ is a cycle which represents the relative fundamental class of $M_{g}$. (Note in passing that this construction may be exploited to prove the inequality $\|M, \partial M\| \leqslant \sigma(M)$ stated in the introduction, which holds for every 3-manifold $M)$. Since we know that the straightening operator induces the identity on homology, the $\operatorname{cycle~} \operatorname{str}\left(z_{M}^{\mathbb{R}}\right)$ also represents the relative fundamental class of $M_{g}$.

The cycle $\operatorname{str}\left(z_{M}^{\mathbb{R}}\right)$ can be realized as the push-forward of a relative cycle in $C_{n}(|P|, \partial|P| ; \mathbb{R})$ via $\operatorname{str}_{T}$. To see this, we denote by $\hat{\sigma}_{i}: \Delta^{3} \rightarrow|P|$ the singular simplex corresponding to $\sigma_{i}: \Delta^{3} \rightarrow M_{g}, i=1, \ldots, N_{0}$. Here also, the sum of the $\hat{\sigma}_{i}$ 's does not provide in general an integral relative cycle for $(|P|, \partial|P|)$, so we have to recur to the real relative cycle $z_{P}^{\mathbb{R}}=\operatorname{alt}\left(\hat{\sigma}_{1}\right)+$ $\ldots+\operatorname{alt}\left(\hat{\sigma}_{N_{0}}\right)$. By contruction, the chains $\left(\operatorname{str}_{T}\right)_{*}\left(z_{P}^{\mathbb{R}}\right)$ and $\operatorname{str}\left(z_{M}^{\mathbb{R}}\right)$ differ just 
by a linear combination of simplices supported on $\partial M_{g}$. As a consequence, they define the same element of $C_{n}\left(M_{g}, \partial M_{g} ; \mathbb{R}\right)$, so $\left(\operatorname{str}_{T}\right)_{*}\left(z_{P}^{\mathbb{R}}\right)$ is a relative fundamental cycle of $M_{g}$.

Since $P$ is an orientable 3 -dimensional pseudomanifold, every connected component of $\partial|P|$ is a closed orientable surface. If we denote by $[\partial P]$ the sum of the real fundamental classes of the components of $\partial|P|$, then our previous considerations imply that $\left(\operatorname{str}_{T}\right)_{*}([\partial P])$ is equal to the sum of the fundamental classes of the boundary components $\partial_{0} M_{g}, \partial_{1} M_{g}$ of $M_{g}$. In particular, for $i=0,1$, there exist components $\partial_{i}|P|$ of $\partial|P|$ such that the restriction $\left.\operatorname{str}_{T}\right|_{\partial_{i}|P|}: \partial_{i}|P| \rightarrow \partial_{i} M_{g}$ has positive degree. This implies that $g_{i} \geq g$, where $g_{i}$ is the genus of $\partial_{i}|P|$.

As usual, we denote by $t_{j}$ the number of simplices of $P$ with exactly $j$ boundary faces, and we recall that $t_{2}=t_{3}=t_{4}=0$, so that $N_{0}=t_{0}+t_{1}$. Since the $\Delta$-complexity of a closed surface of genus $\bar{g}$ is $4 \bar{g}-2$ we get

$$
t_{1} \geq\left(4 g_{0}-2\right)+\left(4 g_{1}-2\right) \geq 8 g-4 .
$$

Just as in the computations leading to Theorem 4, we now need to bound $t_{0}$ from below. To this aim we exploit Proposition 3.7 with $d=1$. Actually, the proposition is stated for pseudomanifolds associated to integral cycles, but the proof carries through without changes in our present setting of a pseudomanifold $P$ with a map of pairs

$$
\operatorname{str}_{T}:(|P|, \partial|P|) \rightarrow\left(M_{g}, \partial M_{g}\right)
$$

sending the real fundamental class of $(|P|, \partial|P|)$ to the real fundamental class of $\left(M_{g}, \partial M_{g}\right)$. Keeping notations and terminology from above, we denote by $E_{\text {nice }}$ the number of nice edges of $\partial|P|$, and we recall that $t_{0} \geq E_{\text {nice }} / 2$. Since $M_{g}$ has two boundary components, Proposition 3.7 implies that

$$
t_{0} \geq \frac{E_{\text {nice }}}{2} \geq \frac{8+\left\|\partial M_{g}\right\|}{4}=2 g .
$$

Putting together this inequality with Inequality (15) we get

$$
N \geq N_{0}=t_{0}+t_{1} \geq 2 g+8 g-4=10 g-4,
$$

which concludes the proof.

Proof of Corollary 6. Using that the stable $\Delta$-complexity bounds the simplicial volume from above and applying Theorem 4 , we have the inequalities

$$
\sigma_{\infty}\left(M_{g}\right) \geq\left\|M_{g}, \partial M_{g}\right\| \geq \frac{5}{4}\left\|\partial M_{g}\right\|=10(g-1) .
$$

It remains to see that the stable $\Delta$-complexity of $M_{g}$ is smaller than or equal to $10(g-1)$. For every $d \geq 2$ the manifold $M_{g}$ admits a covering of degree $d$ whose total space is homeomorphic to $M_{g^{\prime}}$, where $g^{\prime}=d(g-1)+1$. By Theorem 5, this implies that

$$
\sigma_{\infty}\left(M_{g}\right) \leq \frac{\sigma\left(M_{d(g-1)+1}\right)}{d} \leq \frac{10 d(g-1)+6}{d}=10(g-1)+\frac{6}{d} .
$$


Since $d$ is arbitrary, the corollary is proved.

\section{REFERENCES}

$\left[\mathrm{BBF}^{+} 14\right]$ M. Bucher, M. Burger, R. Frigerio, A. Iozzi, C. Pagliantini, and M. B. Pozzetti, Isometric properties of relative bounded cohomology, J. Topol. Anal. 6 (2014), no. 1, 1-25.

[BBI13] M. Bucher, M. Burger, and A. Iozzi, A dual interpretation of the GromovThurston proof of Mostow rigidity and volume rigidity for representations of hyperbolic lattices, Trends in Harmonic Analysis, Springer-Verlag Italia, 2013, pp. $47-76$.

[BK08] M. Bucher-Karlsson, The simplicial volume of closed manifolds covered by $\mathbb{H}^{2} \times$ $\mathbb{H}^{2}$, J. Topol. 1 (2008), 584-602.

[FFM12] S. Francaviglia, R. Frigerio, and B. Martelli, Stable complexity and simplicial volume of manifolds, J. Topol. 5 (2012), 977-1010.

[FM11] K. Fujiwara and J. K. Manning, Simplicial volume and fillings of hyperbolic manifolds, Algebr. Geom. Topol. 11 (2011), 2237-2264.

[FP10] R. Frigerio and C. Pagliantini, The simplicial volume of hyperbolic manifolds with geodesic boundary, Algebr. Geom. Topol. 10 (2010), 979-1001.

[Fra04] S. Francaviglia, Hyperbolic volume of representations of fundamental groups of cusped 3-manifolds, Int. Math. Res. Not. 9 (2004), 425-459.

[Gro82] M. Gromov, Volume and bounded cohomology, Inst. Hautes Études Sci. Publ. Math. 56 (1982), 5-99.

[Hat02] A. Hatcher, Algebraic topology, Cambridge University Press, Cambridge, 2002.

[JJST] W. Jaco, J. Johnson, J. Spreer, and S. Tillmann, Bounds for the genus of a normal surface, arXiv:1411.6413.

[JR] W. Jaco and H. Rubinstein, Layered-triangulations of 3-manifolds, arXiv:math/0603601.

[JRT09] W. Jaco, H. Rubinstein, and S. Tillmann, Minimal triangulations for an infinite family of lens spaces, J. Topol. 2 (2009), 157-180.

[Jun97] D. Jungreis, Chains that realize the Gromov invariant of hyperbolic manifolds, Ergod. Th. Dynam. Sys. 17 (1997), 643-648.

[Kue03] T. Kuessner, Multicomplexes, bounded cohomology and additivity of simplicial volume, arXiv:math/0109057, 2003.

[Mar06] B. Martelli, Complexity of 3-manifolds, Spaces of Kleinian groups, London Math. Soc. Lec. Notes Ser., vol. 329, 2006, pp. 91-120.

[MP01] B. Martelli and C. Petronio, 3-manifolds having complexity at most 9, Exp. Math. 10 (2001), 207-237.

[MT77] J. Milnor and W. Thurston, Characteristic numbers for three-manifolds, Enseignment Math. 23 (1977), 249-254.

[Rat94] J. G. Ratcliffe, Foundations of hyperbolic manifolds, Graduate Texts in Mathematics, Springer-Verlag, New York, 1994.

[Thu79] W. P. Thurston, The geometry and topology of 3-manifolds, Princeton, 1979, mimeographed notes.

[Thu97] _ Three-dimensional geometry and topology, Princeton Mathematical Series, vol. 35, Princeton University Press, Princeton, NJ, 1997, edited by S. Levy.

Section de Mathématiques, 2-4 Rue du Lièvre, Case postale 64, 1211 Genève 4, Suisse

E-mail address: michelle.bucher-karlsson@unige.ch 
Dipartimento di Matematica, Università di Pisa, Largo B. Pontecorvo 5, 56127 Pisa, ITALY

E-mail address: frigerio@dm.unipi.it

Department Mathematik, ETH Zentrum, 8092 Zürich

E-mail address: cristina.pagliantini@math.ethz.ch 